\section{The Italian strategy to fight illegal treatment with growth promoters: Results of the 2017-2019 histological monitoring plan}

\author{
Marzia Pezzolato, Elisa Baioni, \\ Cristiana Maurella, Alessandro \\ Benedetto, Elena Biasibetti, \\ Elena Bozzetta \\ CIBA, Istituto Zooprofilattico \\ Sperimentale del Piemonte, Liguria e \\ Valle d'Aosta, Turin, Italy
}

\begin{abstract}
The Histological Control Plan has been introduced in Italy in 2008 as an indirect monitoring tool of illicit administration of sexual hormones and corticosteroids in bovine. Analysis of 2008-2016 results permitted to draw a new plan targeting risk category. This work presents the results of the histopathological monitoring plan that was carried out from 2017 to 2019. The overall prevalence of samples suspected of treatment with corticosteroid was $11.3 \%$ [ $95 \%$ confidence interval (CI) 6.6-17.8] in 2017; $10.2 \%$ (95\% CI 6.6-16.9) in 2018 and $8.9 \%$ (95\% CI 4.6-15.4) in 2019. The overall prevalence of samples suspected of treatment with sexual hormones was $2.3 \%$ (95\% CI $0.5-6.6)$ in $2017 ; 6.2 \%(95 \%$ CI 2.7-11.8) in 2018 and $12.4 \%$ (95\% CI 7.419.1) in 2019. Although not targeting and measuring specific molecules, this strategy allows to verify the trend of illicit treatments and identify farms to be submitted to further check.
\end{abstract}

\section{Introduction}

Growth promoting agents in food producing animals are strictly forbidden in the European Union (EU) by the Council Directive 96/23/EC (Directive 96/23/EC). The term 'Illegal treatment' means 'the use of unauthorized substances or products or the use of substances or products authorized under Community legislation for purposes or under conditions other than those laid down in Community legislation. The prohibition is in place to protect consumers', in fact the ingestion or consumption of residue and or metabolites of unauthorized drugs poses a serious risk to consumers' health.
Every year the European Union focuses its efforts in fighting the illegal use of substances for growth-promoting purposes, by implementing Communitarian Residues Control Plans to monitor the presence of hormonal and veterinary drugs residues in food and feedstuffs (Directive 96/23/EC 1). Despite the intense official control activity, in the 354,517 targeted sample reported to the European Community by the 28 Member States, the percentage of non-compliant samples recovered in 2018 was $0.30 \%$ (EFSA, 2020). Particularly in the group of sexual hormones (A3) and corticosteroids (B2F), only $0.16 \%$ and $0.15 \%$ samples respectively were found to be noncompliant in cattle (EFSA, 2020).

From the analysis of data of National Residue Control Plans (NRCPs), in 2013 the European Agency for Food Safety (EFSA) underlined the inefficiency of the screening strategy implemented in the Official Monitoring Plans, suggesting and encouraging the development and use of biologically based methods, in order to improve the effectiveness of the official screening steps of Residues Control (EFSA Scientific Opinion, 2013).

To date this recommendation has been adopted only by the scientific community through the development of untargeted methods to expose ongoing illegal treatments (Dervilly-Pinel et al., 2018) while the legislation in force is not yet adequate. To fill this gap, National Reference Laboratories are involved in the revision of the legislation on residues of pharmacologically active substances controls. In 2008 the Italian Ministry of Health introduced the histological plan as a complementary strategy of control. The objective of this monitoring activity was to verify at national level whether the samples from the slaughterhouses exceeded a predefined prevalence threshold level for each illicit treatment subject to surveillance: corticosteroids and sexual hormones $(\mathrm{P}=15 \%)$.

To achieve this goal, histological analyses were performed on a statistically significant number of slaughtered animals by the Experimental Zooprophylactic Institutes (IIZZSS) official laboratories. The histopathological examination can detect lesions induced by sexual hormones and glucocorticoids in bovines target organs (i.e., sexual accessory glands and thymus) (Groot et al., 2007, Imbimbo et al., 2012). Sexual hormones are known to cause squamous metaplasia in the epithelium of sexual accessory glands (Pezzolato et al., 2013) while glucocorticoid cause atrophy in thoracic thymus cortex (Bozzetta et al., 2011;
Correspondence: Elena Biasibetti, Istituto Zooprofilattico Sperimentale del Piemonte, Liguria e Valle d'Aosta, Via Bologna 148, 10154, Turin, Italy.

Tel.: 0112686362 - Fax: 0112686362

E-mail: elena.biasibetti@izsto.it

Key words: Growth promoters; Histological monitoring; Bovine.

Acknowledgments: The authors would like to thank the net of Istituti Zooprofilattici Sperimentali for the continuous collaboration for the PNR application. The authors are also grateful to the public veterinary service and the veterinary practitioners.

Contributions: The authors contributed equally.

Conflicts of interest: The authors declare no conflict of interest

Funding: This research received no external funding. The data of this work represent the routine work of Centro di Referenza Nazionale per le Indagini Biologiche sugli Anabolizzanti Animali (CIBA) activity.

Received for publication: 22 March 2021

Revision received: 14 September 2021.

Accepted for publication: 26 October 2021.

This work is licensed under a Creative Commons Attribution-NonCommercial 4.0 International License (CC BY-NC 4.0).

(C) Copyright: the Author(s), 2021

Licensee PAGEPress, Italy

Italian Journal of Food Safety 2021; 10:9775 doi:10.4081/ijfs.2021.9775

Zanardello et al., 2018). These changes represent biomarkers that persist far longer than the negativization of the residues in the official matrices analyzed by analytical methods included in the NRCPs (Richelmi et al., 2017). These histopathological findings proved to be reliable markers of treatment. In 2008 they were validated to be applied in the histological plan and became part of the Standard Operating Procedures (SOPs) shared among the IIZZSS.

In order to improve the efficiency of the national monitoring plan, data from histological samples collected between 2008 and 2016 have been analyzed to determine on which high risk categories future checks should be focused.

The aim of this study is to present the results of the histopathological monitoring plan carried out from 2017 to 2019 to expose the illicit administration of sexual hormones and corticosteroids in in food producing bovines. 


\section{Materials and methods}

\section{7-2019 Monitoring Control Plans}

The objective of this monitoring activity was to verify at national level whether the batches from the slaughterhouses exceeded a predefined prevalence threshold level for each illicit treatment subject to surveillance: corticosteroids and sexual hormones $(\mathrm{P}=15 \%)$. According to this design, samples of thymus and sexual accessory glands of beef cattle and veal calves were analyzed by histopathology according to the SOPs in use in the histopathological laboratories of IIZZSS.

Results were reviewed in order to obtain further information's regarding highrisk categories to be accounted for in the sampling design of the new National Monitoring Plan of the present study.

To confirm that the observed prevalence of sexual hormones illegal treatments in veal calves is equal or above the $10 \%$ threshold, 147 batches (first stage) were calculated to be sampled and at least 20 batches had to be evaluated as not compliant.

The number of animals (second stage) estimated to be sampled within each batch was equal to: all animals in the case of batches consisting of less than 5 animals; 5 in the case of batches consisting of 5 to 7 animals; 6 in batches consisting of more than 7 animals.

To verify that the observed prevalence of corticosteroid illegal treatments in beef cattle is equal or above the $13 \%$ threshold, 104 batches were calculated to be sampled and at least 14 batches had to be evaluated as not compliant.

Based on these criteria, the number of animals estimated to be sampled within each batch was equal to all animals in the case of batches consisting of less than 5 animals; 5 in the case of batches consisting of 5 to 7 animals; 6 in batches consisting of more than 7 animals.

The objective of the new monitoring plan was to verify whether at national level the batches sent to the slaughterhouse exceed a predefined prevalence threshold level for each illicit treatment subject to and veal calves $(\mathrm{P}=13 \%$ for beef, $\mathrm{P}=10 \%$ for veal). The activity from 2017 to 2019 was planned on veal calves to search for illicit treatments with sexual hormones and on beef cattle to expose corticosteroid treatments. A two-stage sampling was used to demonstrate a treatment free status respectively from sexual hormones in veal calves and corticosteroids in beef cattle. The first stage consisted in selecting a sample of batches; the second stage involved selecting a sample of animals from each batch of stage one. Parameters used to estimate the sample size to demonstrate the absence of treatment with sexual hormones in veal calves (sampling and analysis of sexual accessory glands: prostate and urethral bulb glands) are shown in Table 1.

The cut-point number of suspected animals to demonstrate batch sexual hormones treatment free status, calculated using imperfect tests and allowing for small populations, is shown in Table 2: one batch was considered suspected if a certain number of animals equal or greater than the cut-point number was found in the random sample collected from the batch.

Parameters used to estimate the sample size to demonstrate corticosteroids treatment free status in beef cattle (thymus sample and analysis) are shown in Table 1.

The cut-point number of suspected anisurveillance, separately for the beef cattle

mals to demonstrate batch freedom from corticosteroid treatment are the same reported in Table 2.

\section{Sampling process}

Sampling was performed by official veterinarians in all Italian regions: a training course was organized to ensure that the sampling criteria were adhered by all field workers. To guarantee the representativeness of the sampling the operators were asked to perform a random sampling, avoiding criteria in the choice of batches, and applying an even geographical and temporal distribution of the sampling during the year. The thoracic thymus and sexual accessory glands (prostate and bulbo urethral glands) were collected at the slaughterhouse, fixed in neutral buffered formalin, and sent to the laboratories of the IIZZSS to be analyzed according to the dedicated SOPs.

\section{Histopathology}

Samples were trimmed, routinely processed, embedded in paraffin wax and serially sectioned at $3+/-2 \mu \mathrm{m}$ for the histological evaluation. Slides were stained with Hematoxylin and Eosin and observed by optic microscopy (ZEISS Axio Scope.A1, Köln, Germany)

Samples were analyzed to highlight severe atrophy (Richelmi et al., 2017) (Figure 1A) in thymus and metaplasia in sexual accessory glands (Figure 1B) (Pezzolato et al., 2013) according to the SOP criteria in place.

Table 1. Parameters used to estimate the sample size for veal calves' population.

\begin{tabular}{lcc} 
Parameter & First stage (batches) $\%$ & Second stage (animals) \% \\
Confidence level & 95 & 95 \\
Sensitivity & 95 & 90 \\
\hline Specificity & 90 & 80 \\
Expected prevalence & 10 & 80 \\
\hline Statistical power & 90 & 90 \\
\hline
\end{tabular}
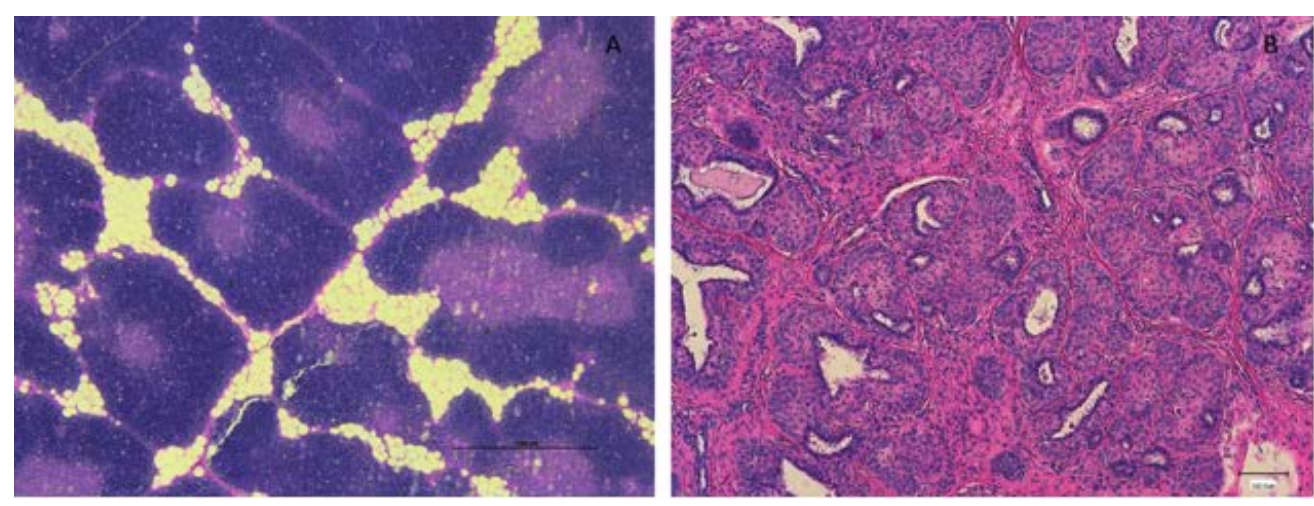

Figure 1. Thymus samples of veal calves suspect for corticosteroid treatment with severe atrophy. Haematoxylin-eosin staining (A). Sexual accessory gland of veal calves suspects for sex steroid treatment showed severe metaplasia. Haematoxylin-eosin staining (B). 
The data on the presence of lesions for each animal were recorded into a database (DB).

\section{Statistical analysis}

Results registered in the DB were used to calculate the total percentage of noncompliances. An extension of the Wilcoxon rank-sum as developed by Cuzick (1985) was used to verify a statistically significant variation of treatment over years. All analysis were performed with Stata 16.1 (StataCorp LLC, 4905 Lakeway Drive College Station, Texas 77845-4512 USA).

\section{Results}

According to the results from the 20082016 plans, a new plan was designed and a sample size of 147 batches was calculated in order to increase the performance of the surveillance system.

The total number of beef cattle batches sampled for corticosteroids was 141 in 2017, 127 in 2018 and 123 in 2019 for a total of 340,325 and 304 beef cattle analyzed respectively each year.

The total number of veal calves' batches sampled for sexual hormones was 129 in 2017, 130 in 2018 and 137 in 2019 for a total of 426, 386 and 390 veal calves analyzed respectively each year.

The overall prevalence of treatment with corticosteroid was $11.3 \%$ (95\% confidence interval (CI) 6.6-17.8) in 2017; $10.2 \%(95 \%$ CI $6.6-16.9)$ in 2018 and $8.9 \%$ (95\% CI 4.6-15.4) in 2019.

Suspected batches for corticosteroid treatment are reported in Table 3.

The overall prevalence of treatment with sexual hormones is $2.3 \%(95 \%$ CI 0.5 $-6.6)$ in $2017 ; 6.2 \%$ (95\% CI $2.7-11.8)$ in 2018 and $12.4 \%$ (95\% CI 7.4-19.1) in 2019.
Suspected batches for sexual hormones treatment are reported in Table 4.

The tests for trend were not statistically significant (Figure 2).

\section{Discussion and Conclusions}

Application of growth promoting agents like steroid hormones or anabolic hormones cocktails are well known to increase muscle mass in beef cattle. The main purpose is to reduce lipid mass and increase muscle tissue. These agents promote overall tissue growth, by increasing growth rates, improve the conversion of nitrogen in feed into muscle protein and hence increase the amount of lean and valuable meat in the carcass (Stephany, 2010).

The application of any growth promoting agents is strictly forbidden in livestock production within the EU. Estrogens are known to cause cancer in humans while androgens are related to the development of different neoplasms in animals. Corticosteroids are dangerous for human health, causing in vulnerable people hyperglycemia, diabetes, high blood pressure and maximizing risk of infection (Kavanaugh and Wells, 2014).

At present, these growth promoting drugs are screened within an EU control program, using sensitive immunologicalbased test systems (e.g., ELISA, enzymelinked immunosorbent assays) or chromatographic methods in combination with mass spectrometry (Meyer and Hoffmann, 1987; Meyer et al., 1991; Scippo et al., 1994). Using these established methods, newly designed anabolic drugs cannot be identified until their chemical structure is known, or a specific and high affinity antibody is available for the immune tests. A further problem is the identification of a single low dosage drug mixed in a drug cocktail. These undefined cocktails due to their low concentrations are barely detectable and difficult to quantify in a reproducible manner using conventional detection methods (Cantiello et al., 2007). Therefore, development of new screening methods is required to detect the use of such substances, independently of their structure and/or concentration (Nebbia et al., 2001).

The determination of microscopical changes caused using growth promoting and anabolic substances based on the analysis of target organs changes is a simple but effective long known method (Groot et al., 2007, De Maria et al., 2009; Cannizzo et al., 2001), to detect permanent changes related to these illegal treatments. Thanks to the process of validation of few reliable

Table 2. The cut-point number of suspected animals to demonstrate batch freedom from treatment.

\begin{tabular}{|cc|} 
Sample of animals & Cut-point number \\
\hline 1 & 1 \\
2 & 2 \\
\hline 3 & 3 \\
\hline 4 & 3 \\
\hline 5 & 3 \\
\hline 6 & 3 \\
\hline
\end{tabular}

Table 3. Suspected batches for corticosteroid treatment and sex steroid treatments.

\begin{tabular}{ccccccc} 
& Batches sampled & $\begin{array}{c}\text { Corticosteroid } \\
\text { Batches suspected }\end{array}$ & $\%$ & Batches sampled & $\begin{array}{c}\text { Sexual hormones } \\
\text { Batches suspected }\end{array}$ \\
2017 & 141 & 16 & 11.3 & 129 & 3 & 2.3 \\
2018 & 127 & 13 & 10.2 & 130 & 9.2 \\
\hline 2019 & 123 & 11 & 8.9 & 137 & 17 & 12.4 \\
\hline
\end{tabular}

Table 4. Parameters used to estimate the sample size for beef cattle population.

\begin{tabular}{|c|c|c|}
\hline Parameter & First stage (batches) \% & Second stage (animals) \% \\
\hline Confidence level & 95 & 95 \\
\hline Sensitivity & 95 & 90 \\
\hline Specificity & 90 & 80 \\
\hline Expected prevalence & 13 & 80 \\
\hline Statistical power & 90 & 90 \\
\hline
\end{tabular}




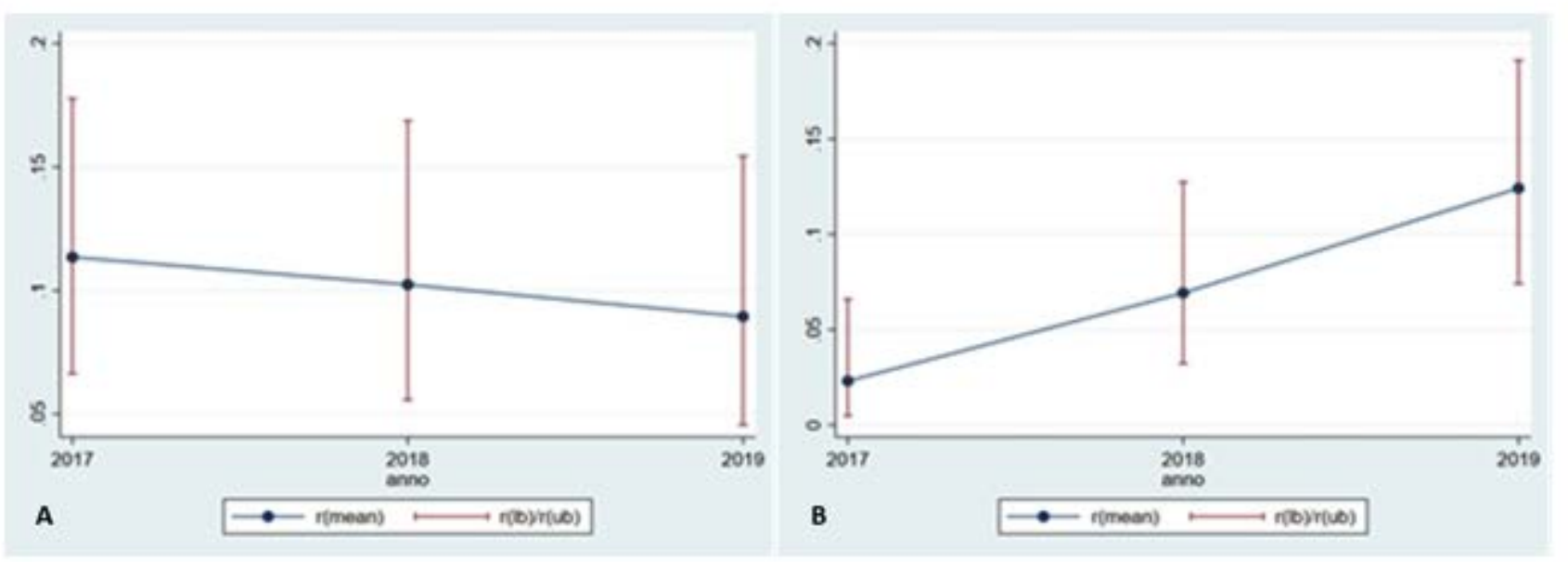

Figure 2. Trend of prevalence respectively for corticosteroid treatments in beef cattle (A) and for sexual steroid treatments in veal calves (B). Vertical bars show confidence interval width.

microscopic markers, a monitoring plan for both sexual hormones and corticosteroids could be drawn in Italy in 2008; the efficacy and effectiveness of this plan has recently improved. This is currently applied in the frame of a strategy to highlight farms to be controlled with standard analytical methods in order to fight the misuse of anabolic agents in cattle.

The results achieved by the monitoring histological plan applied from 2017 to 2019 are in contrast with those reported by the Italian NRCP and demonstrate that the phenomenon of illicit treatments is far from being eradicated (EFSA, 2020). Even if histology is not able to identify the administered treatment, the results obtained by the monitoring plan can help directing official controls. In the past few years most of the farms selected through the histological plan have proved to be noncompliant (data not shown) during official controls.

This study also highlights possible consumers' health risks as illicit cattle treatment may result in illegal/dangerous residues in meat.

In conclusion this work shows how monitoring plans based on an untargeted method can support the fight to drug abuse in farm animals. Due to the rather homogenous situation regarding veterinary drugs residues all over European countries, we can expect that the adoption of alternative strategies suggested by EFSA could disclose the real prevalence of illegal treatments and help to address official controls.

\section{References}

Bozzetta E, Pezzolato M, Maurella C,
Varello K, Richelmi GB, Draisci R, Ferranti C, D'Angelo, A, Caramelli M, 2011. Development of an enhanced histopathological approach to detect low-dose dexamethasone illicit treatment in veal calves. Food Addit Contam Part A Chem Anal Control Expo Risk Assess 28:1187-92.

Cannizzo FT, Capra P, Divari S, Ciccotelli V, Biolatti B, Vincenti M, 2011. Effects of low-dose dexamethasone and prednisolone long term administration in beef calf: chemical and morphological investigation. Anal Chim Acta 700:95104.

Cantiello M, Carletti M, Cannizzo FT, Nebbia C, Bellino C, Pié S, Oswald IP, Bollo E, Dacasto M, 2007. Effects of an illicit cocktail on serum immunoglobulins, lymphocyte proliferation and cytokine gene expression in the veal calf. Toxicology 242:39-51.

Council Directive 96/23/EC (OJ L125, p10, 23/05/1996) of 29 April 1996 on measures to monitor certain substances and residues in live animals and animal products. Brussels, Belgium: European Commission, 1996.

Cuzick J, 1985. A Wilcoxon-type test for trend. Stat Med 4:87-90.

De Maria R, Divari S, Goria M, Bollo E, Cannizzo FT, Olivero M, Barbarino G, Biolatti B, 2009. 17beta-oestradiolinduced gene expression in cattle prostate: biomarkers to detect illegal use of growth promoters. Vet Rec 164:459-64.

Dervilly-Pinel G, Royer AL, Bozzetta E, Pezzolato M, Herpin L, Prevost S, Le Bizec B, 2018. When LC-HRMS metabolomics gets ISO17025 accredit- ed and ready for official controls application to the screening of forbidden compounds in livestock. Food Addit Contam Part A Chem Anal Control Expo Risk Assess 35:1948-58.

EFSA, 2020. Technical report. Report for 2018 on the results from the monitoring of veterinary medicinal product residues and other substances in live animals and animal products. EFSA Supporting Publications 17:3.

EFSA Scientific Opinion, 2013. Guidance on methodological principles and scientific methods to be taken into account when establishing Reference Points for Action (RPAs) for non-allowed pharmacologically active substances present in food of animal origin. EFSA Journal 11:3195.

Groot MJ, Ossenkoppele JS, Bakker R, Pfaffl MW, Meyer HHD, Nielen MWF, 2007. Reference histology of veal calf genital and endocrine tissues - an update for screening on hormonal growth promoters. Vet Med Sci 54:23846.

Imbimbo P, Castigliego L, Armani A Biolatti B, Cannizzo FT, Gianfaldoni D, Guidi AA, 2012. Histologic study on, growth promoter target organs of, slaughtered beef in Molise Region (Italy). J Vet Med Sci 74:1253-9.

Kavanaugh A, Wells AF, 2014. Benefits and risks of low-dose glucocorticoid treatment in the patient with rheumatoid arthritis. Rheumatology (Oxford) 53:1742-51.

Meyer HH, Hoffmann S, 1987. Development of a sensitive microtitration plate enzyme-immunoassay for the anabolic steroid trenbolone. Food Addit 
Contam 4:149-60.

Meyer HH, Rinke L, Duersch I, 1991. Residue screening for the beta-agonists clenbuterol, salbutamol and cimaterol in urine using enzyme immunoassay and high-performance liquid chromatography. J Chromatogr B Biomed Sci Appl 56:551-6.

Nebbia C, Urbani A, Carletti M, Gardini G, Balbo A, Bertarelli D, Girolami F, 2001. Novel strategies for tracing the exposure of meat cattle to illegal growthpromoters. Vet J 189:34-42.

Pezzolato M, Richelmi GB, Maurella C, Pitardi D, Varello K, Caramelli M, Bozzetta E, 2013. Histopathology as a simple and reliable method to detect $17 \beta$-oestradiol illegal treatment in male calves. Food Addit Contam Part A Chem Anal Control Expo Risk Assess 30:1096-9.

Richelmi GB, Maurella C, Pezzolato M, Botta M, Varello K, Pitardi D, Baioni E, Bellino C, D'Angelo A, Caramelli M, Bozzetta E, 2017. Thymus atrophy is an efficient marker of illicit treatment with dexamethasone in veal calves: Results from a triennial experimental study. Res Vet Sci 113:67-72.

Scippo ML, Degand G, Duyckaerts A, Maghuin-Rogister G, Delahaut P, 1994. Control of the illegal administration of natural steroid hormones in the plasma of bulls and heifers. Analyst 119: 263944.

Stephany RW, 2010. Hormonal growth promoting agents in food producing animals. Handb Exp Pharmacol 195:35567.

Zanardello C, Pezzolato M, Capello K, Botta M, Baioni E, Mutinelli F, Vascellari M, Bozzetta E, 2018. Immunohistochemistry by anti-cleavedLamin A: an improved approach to tackle the misuse of glucocorticoids in cattle. Food Addit Contam Part A Chem Anal Control Expo Risk Assess 35:2120-2127. 